\title{
Antibiotic Tetrocarcin-A Down-regulates JAM-A, IAPs and Induces Apoptosis in Triple-negative Breast Cancer Models
}

\author{
SRI HARIKRISHNA VELLANKI ${ }^{1}$, RODRIGO G.B. CRUZ ${ }^{1}$, CATHY E. RICHARDS ${ }^{1}$, \\ YVONNE E. SMITH $^{1}$, LANCE HUDSON $^{1}$, HANNE JAHNS $^{2}$ and ANN M. HOPKINS ${ }^{1}$ \\ ${ }^{1}$ Department of Surgery, Royal College of Surgeons in Ireland, Dublin, Ireland; \\ ${ }^{2}$ Pathobiology Section, School of Veterinary Medicine, University College Dublin, Dublin, Ireland
}

\begin{abstract}
Background/Aim: Triple-negative breast cancers $(T N B C)$ lack expression of three important receptors, and have limited treatment options. High expression of junctional adhesion molecule-A (JAM-A) has been linked with aggressive tumor phenotypes including TNBC. This study aimed to evaluate the bioactivity of a JAM-A-downregulating compound, Tetrocarcin-A, in TNBC. Materials and Methods: TNBC cell viability, colony formation and xenograft growth were examined in Tetrocarcin-A-treated HCC 38 human cells, 4 T1 mouse cells or patient-derived primary cells. Protein expression of cell fate signaling effectors was examined by immunoblotting (versus transient JAM-A gene silencing). Apoptotic pathways were investigated in parallel. Results: Tetrocarcin-A reduced $T N B C$ cell viability in vitro and in an in ovo/semi-in vivo xenograft model. Tetrocarcin-A-induced JAM-A downregulation and reduced ERK phosphorylation, followed by c-FOS phosphorylation on its transcription-regulating residue, which down-regulated several inhibitor of apoptosis (IAP) proteins and induced caspase-dependent intrinsic pathway of apoptosis. Conclusion: Tetrocarcin-A merits further investigation as a novel anti-tumor agent in TNBC.
\end{abstract}

Triple-negative breast cancers (TNBC) are so-called because they lack expression of the estrogen receptor, progesterone receptor and human epidermal growth factor receptor-2. Of the many sub-classifications of breast cancer, TNBCs remain among the most aggressive (1). Reflecting the lack of cell surface markers, treatment options for TNBC are usually limited to conventional chemotherapies rather than molecular

Correspondence to: Dr. Ann M. Hopkins, Royal College of Surgeons in Ireland, RCSI Smurfit Building, Beaumont Hospital, Dublin 9, Ireland. Tel: +353 18093858, e-mail: annhopkins@rcsi.ie

Key Words: Triple negative breast cancer, Tetrocarcin-A, junctional adhesion molecule-A, apoptosis, chorionic allantoic membrane assay, natural compounds. targeted therapies. Thus, there is an ongoing need to identify new reagents with potential bioactivity in TNBC.

Junctional Adhesion Molecule-A (JAM-A) is an adhesion protein which plays important physiological roles in many tissues; but whose high expression has been correlated with aggressive disease and poor outcome in breast cancer patients $(2,3)$. Studies have also shown that JAM-A-null mice form smaller tumors than JAM-A-positive mice, and that blocking JAM-A in 4T1 TNBC mouse mammary cells reduces growth and increases apoptosis (3). Following screening of a natural compound library from the National Cancer Institute Developmental Therapeutics Program (dtp.cancer.gov) using JAM-A-overexpressing versus control cells, the antibiotic Tetrocarcin-A was identified as a compound that downregulates JAM-A while inhibiting cell proliferation and inducing apoptosis in hormone receptor-positive breast cancer cells (4). Tetrocarcin-A was originally isolated from Micromonospora chalcea subsp. Kazunoensis (5), and is principally active against Gram-positive bacteria but, has been also shown to inhibit $\mathrm{AKT}$ (6) and $\mathrm{Bcl} 2$ and induce apoptosis in cancer cells (7). However signaling events upstream of AKT or Bcl2 are not known. Herein, it is shown that Tetrocarcin-A-induced down-regulation of JAM-A expression is associated with reduced TNBC cell viability by a pathway involving reduced c-FOS phosphorylation and the consequent down-regulation of IAPs. Since TNBC tumors are difficult to treat, the capacity of Tetrocarcin-A to remove an endogenous blockade on apoptosis merits comprehensive investigation of its potential value in in TNBC.

\section{Materials and Methods}

Cell lines. TNBC cell lines HCC38 (human) and 4T1 (mouse) were cultured in RPMI medium (Sigma-Aldrich, Arklow, Ireland) supplemented with $10 \%$ foetal bovine serum, $100 \mathrm{U} / \mathrm{ml}$ penicillin and $100 \mu \mathrm{g} / \mathrm{ml}$ streptomycin. Human breast tumour primary cell cultures were generated and grown as described (8).

Alamar Blue cell growth assays. Cells were plated in triplicate in 96-well plates $(5,000 /$ well $)$ and treated $24 \mathrm{~h}$ later with the indicated 
concentrations of Tetrocarcin-A or equivalent volume of vehicle control $(0.01 \% \mathrm{v} / \mathrm{v}$ DMSO). Cells transiently silenced for JAM-A expression (below) were similarly assayed. At the indicated time points Alamar Blue solution was added to each well and incubated for $3 \mathrm{~h}$ at $37^{\circ} \mathrm{C}$. Colour intensity was spectrometrically quantified at $580-610 \mathrm{~nm}$ on a VICTOR ${ }^{\mathrm{TM}}$ X3 Multilabel Reader (Perkin Elmer, Waltham, MA, USA).

Colony forming assays. 4T1 cells were plated in 6-well plates and treated after $24 \mathrm{~h}$ with $2.5 \mu \mathrm{M}$ Tetrocarcin-A or vehicle $(0.01 \% \mathrm{v} / \mathrm{v}$ DMSO). Drug-free medium was added $24 \mathrm{~h}$ later, and cells stained on day 10 with $0.6 \%(\mathrm{w} / \mathrm{v})$ crystal violet and photographed by phase contrast microscopy.

Transfections. HCC38 cells on 6-well plates were transfected with 25 $\mathrm{nM}$ of control siRNA (siGENOME non-targeting siRNA \#1, Dharmacon, Lafayette, CO, USA) or JAM-A siRNA (SASI_Hs01_ 00048785, Sigma-Aldrich) using Lipofectamine-2000 (ThermoFisher Scientific, Waltham, MA, USA) as described (4). Cells were harvested after $72 \mathrm{~h}$ for western blotting, or used in cell growth assays. Alternative JAM-A siRNA constructs replicated our results in key assays (data not shown).

Electrophoresis and western blotting. Following treatment with $2.5 \mu \mathrm{M}$ Tetrocarcin-A or JAM-A silencing, TNBC cells were scraped in $200 \mu \mathrm{l}$ lysis buffer composed of $0.1 \mathrm{M} \mathrm{KCl}, 2.5 \mathrm{mM}$ $\mathrm{NaCl}, 3.5 \mathrm{mM} \mathrm{MgCl} 2,10 \mathrm{mM}$ HEPES pH7.4, $1 \%$ Triton-X100, protease and phosphatase inhibitors (Sigma). Cells were then lysed via trituration, centrifuged at $1,500 \times \mathrm{g}$ for $5 \mathrm{~min}$ at $4^{\circ} \mathrm{C}$ and supernatants stored at $-20^{\circ} \mathrm{C}$. Protein content was quantified via bicinchoninic (BCA) assay (Thermo Scientific), whereupon $20 \mu \mathrm{g}$ protein/lane were subjected to reducing SDS-PAGE, transferred to nitrocellulose membranes at $100 \mathrm{~V}$ for $75 \mathrm{~min}$ and immunoblotted with antibodies to human JAM-A, caspase 9, (BD Biosciences, San Jose, CA, USA), full length caspase-3, pERK1/2, total ERK1/2, cIAP-1, XIAP-2 (Cell Signaling Technologies, Danvers, MA, USA), p-c-FOS-T232, $\beta$-actin (Abcam, Cambridge, UK) and cIAP-2 (R\&D Systems, Abingdon, UK). Image Lab software (Bio-Rad Laboratories, Hertfordshire, UK) was used for densitometric quantification of protein bands, using $\beta$-actin as a loading control. Results of 3 independent blots were graphed along with the standard error of the mean (SEM) values.

Flow cytometry. $4 \mathrm{~T} 1$ cells were plated in 6-well plates and treated $24 \mathrm{~h}$ later with $2.5 \mu \mathrm{M}$ Tetrocarcin-A or vehicle $(0.01 \% \mathrm{v} / \mathrm{v}$ DMSO). Cells were stained using an Annexin V/propidium iodide (PI) kit as described (4). Separately, 4T1 cells were plated in 24-well plates and treated $24 \mathrm{~h}$ later with $2.5 \mu \mathrm{M}$ Tetrocarcin-A or vehicle $(0.01 \% \mathrm{v} / \mathrm{v}$ DMSO). After $1 \mathrm{~h}$ incubation, cells were treated with $50 \mu \mathrm{M}$ ZVADFMK (R\&D Systems) or vehicle control ( $0.01 \%$ v/v DMSO). Cells were stained with PI as per manufacturer's instructions (BD Biosciences) and images acquired on a BD FACS-Calibur flow cytometer (BD Biosciences). For flow cytometric measurement of mitochondrial membrane potential, cells were stained with tetramethylrhodamine methyl ester perchlorate (TMRM) (Sigma-Aldrich).

CAM assay and immunohistochemistry (IHC). Semi-in vivo chorionic allantoic membrane (CAM) assays were performed as described previously $(4,9,10)$. Ethical approval was granted by the RCSI Research Ethics Committee, but an animal licence was not required under EU Directive 2010/63/EU. Briefly, $2 \times 10^{6} 4 \mathrm{~T} 1 \mathrm{TNBC}$ mouse mammary cells were resuspended in $25 \mu \mathrm{l}$ serum-free medium and $25 \mu \mathrm{l}$ Matrigel (BD Biosciences) and implanted on the CAM of fertilized chicken eggs on day 8 of gestation. Tumors were topically treated with $2.5 \mu \mathrm{M}$ Tetrocarcin-A or $0.01 \% \mathrm{v} / \mathrm{v}$ DMSO in $15 \mu \mathrm{l}$ PBS during days 10-13 of gestation. Tumors and surrounding CAM were sampled on day 13 , fixed in $4 \%(\mathrm{w} / \mathrm{v})$ paraformaldehyde, paraffin-embedded and cut into $5 \mu \mathrm{m}$ sections. Sections were deparaffinised in xylene, stained with hematoxylin/ eosin or an antihuman cleaved caspase-3 antibody (Cell Signaling Technologies). Cellular apoptosis was assessed by quantifying the percentage of tumor cells expressing cleaved caspase-3 (based on counting 500 tumor cells in representative areas).

\section{Results}

Tetrocarcin-A inhibited TNBC cell growth in a manner which phenocopies JAM-A silencing. Tetrocarcin-A, identified from a compound library screen, significantly reduced the growth of HCC38 and 4T1 TNBC cells (Figure 1a). It also inhibited the long-term survival of 4T1 mammary cancer cells despite a very brief treatment time (Figure 1b). Furthermore, sublethal doses of Tetrocarcin-A sensitized HCC38 breast cancer cells to the chemotherapeutic drug doxorubicin (data not shown). The indicated concentration of Tetrocarcin-A significantly reduced JAM-A expression levels in HCC 38 cells by $24 \mathrm{~h}$ (Figure 1c); although JAM-A reductions started as early as 10 $\mathrm{h}$ (preliminary data not shown). JAM-A loss by gene silencing phenocopied the negative effects of Tetrocarcin-A on cell viability (Figure 1d). These data are consistent with studies showing that gene silencing or pharmacological inhibition of JAM-A inhibits the proliferation of several cancer cell types $(3,11)$. However the role of JAM-A in TNBC remains unclear; although interrogation of a gene expression dataset [http://kmplot.com/analysis $(12,13)]$ revealed a trend towards poorer recurrence-free survival in TNBC patients exhibiting high expression of the JAM-A gene (F11R; data not shown).

Tetrocarcin-A inhibited pro-survival signaling. Next, signaling downstream of Tetrocarcin-A-induced JAM-A loss was investigated and reductions in the MAPK effector phospho-ERK1/2 in HCC38 TNBC cells mimicking the effect of JAM-A gene silencing were noted (Figure 2a). ERK transcriptionally activates the pro-survival transcription factor c-FOS by phosphorylating it on residues such as Threonine$232(14,15)$. Therefore, the effect of JAM-A down-regulation (induced by either Tetrocarcin-A treatment or JAM-A gene silencing) on the levels of Threonine-232-phosphorylated cFOS was examined. As shown in Figure 2b, either Tetrocarcin-A treatment or JAM-A gene silencing resulted in reduced Threonine-232-phosphorylated c-FOS levels.

As this should reduce c-FOS transcriptional activity, bioinformatics were utilised to identify affected genes. From ChIP-seq data, the Encode transcription factor targets dataset (https://www.encodeproject.org/) suggested the anti-apoptotic 

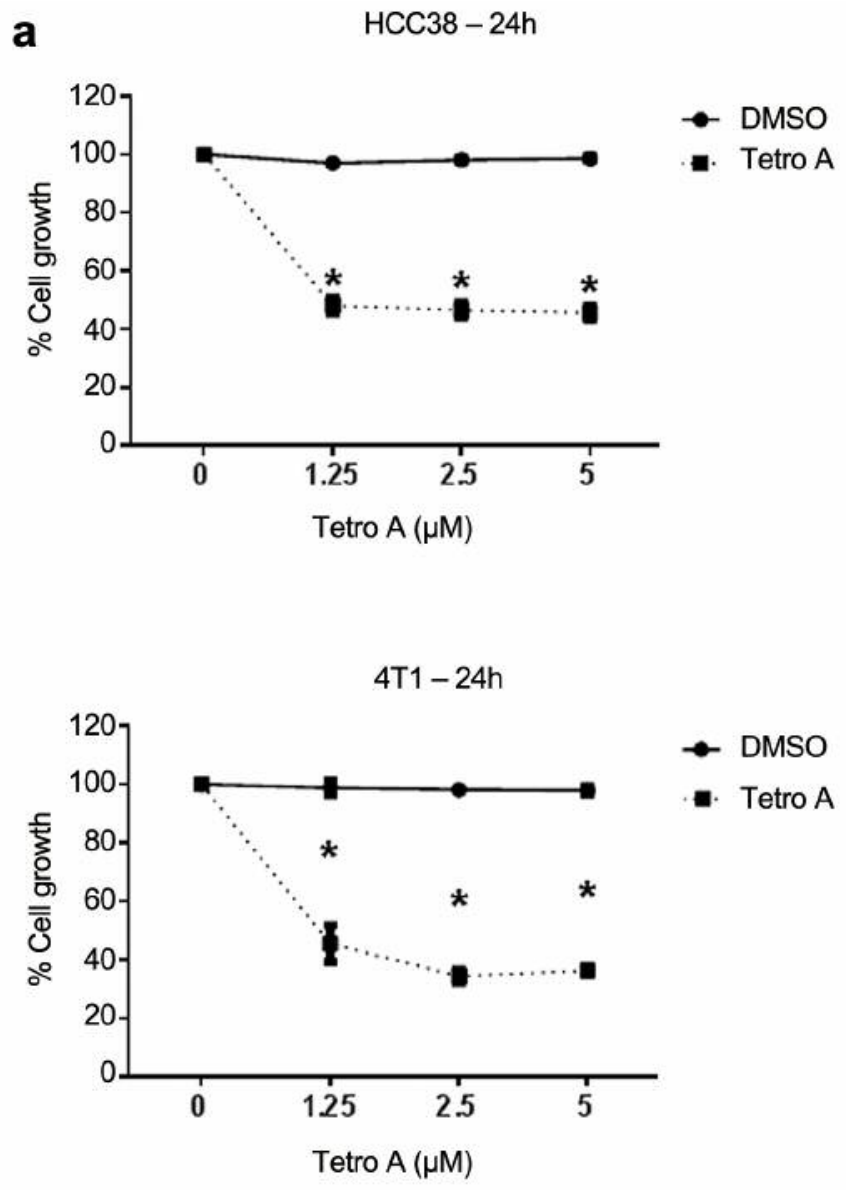

b
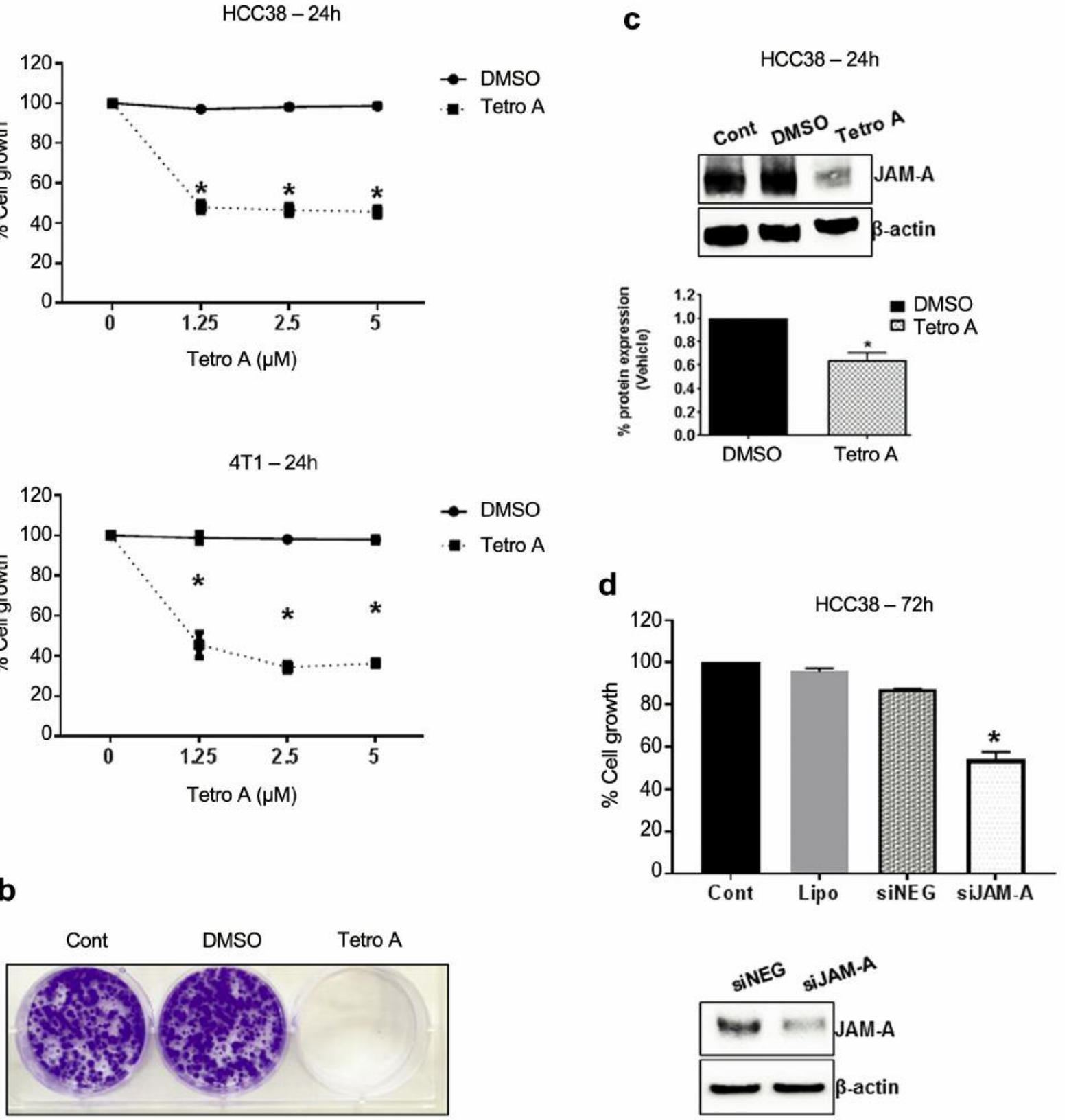

Figure 1. Tetrocarcin-A treatment and JAM-A gene silencing inhibited cell growth of TNBC cells. (a) HCC38 and 4 T1 cells were treated with the indicated concentrations of Tetrocarcin-A or vehicle (0.01\% v/v DMSO). Cell growth was assessed at 24 h by the Alamar Blue assay. (b) $4 T 1$ cells were treated for $24 \mathrm{~h}$ with $2.5 \mu \mathrm{M}$ Tetrocarcin-A or vehicle $(0.01 \% \mathrm{v} / \mathrm{v}$ DMSO). Ten days after compound withdrawal, colonies were stained with crystal violet. (c) HCC38 cells were treated for $24 \mathrm{~h}$ with $2.5 \mu \mathrm{M}$ Tetrocarcin-A or vehicle $(0.01 \% \mathrm{v} / \mathrm{v}$ DMSO), and extracts immunoblotted for JAM$A$ and $\beta$-actin. (d) HCC38 cells were gene-silenced for JAM-A (versus non-targeting sequence, siNEG) and cell viability assessed at 72 h. Error bars refer to SEM of triplicate experiments ( ${ }^{*} p<0.05$ by two-tailed unpaired student's $t$-test; vehicle versus Tetrocarcin-A or siNEG versus siJAM-A).

proteins cIAP-1, cIAP-2 and XIAP as c-FOS targets. This was confirmed by down-regulation of c-IAP1, c-IAP2 and XIAP in HCC38 cells upon Tetrocarcin-A treatment (Figure 3a). Tetrocarcin-A also reduced Bcl-2 and Bcl-xL levels (Figure 3a).
Tetrocarcin-A induced caspase-dependent apoptosis in $T N B C$ cells. The reduction in the levels of anti-apoptotic proteins upon treatment with Tetrocarcin-A suggested the removal of a physiological block on apoptosis. Therefore, the cell death mechanisms induced by Tetrocarcin-A were 
a
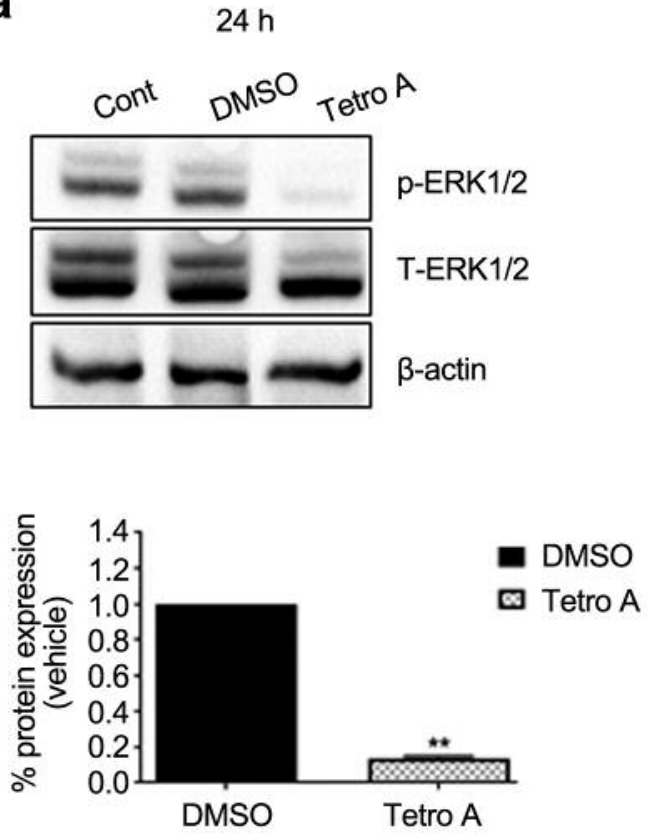

b

$24 \mathrm{~h}$

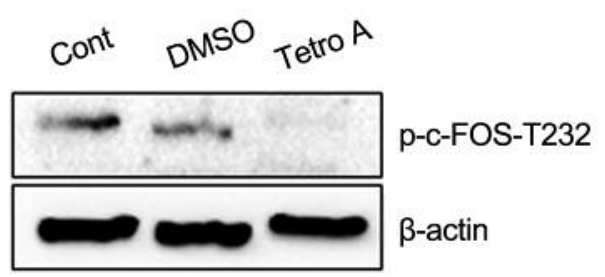

$72 \mathrm{~h}$
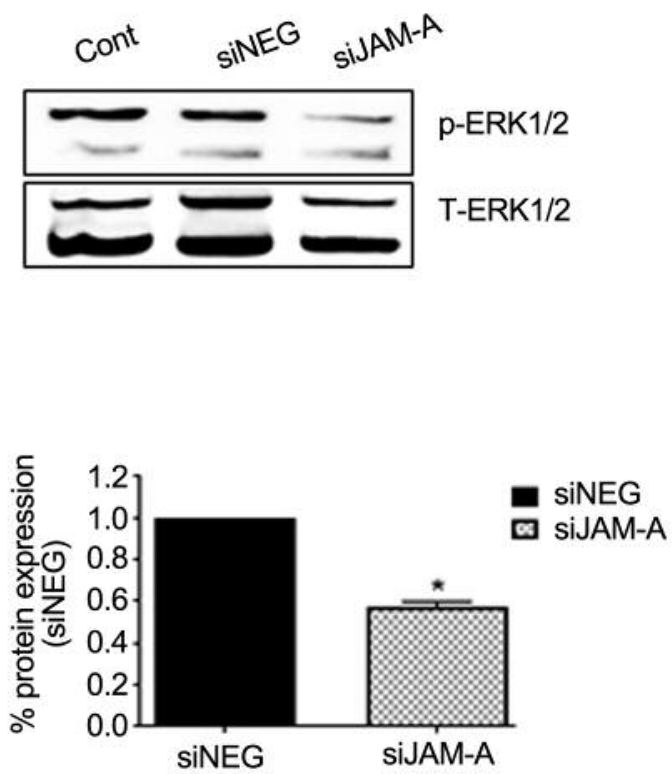

Figure 2. Tetrocarcin-A and JAM-A silencing reduced PERK levels and c-FOS phosphorylation on its transcription-activating residue. HCC38 cells were treated with $2.5 \mu \mathrm{M}$ Tetrocarcin-A or vehicle $(0.01 \% \mathrm{v} / \mathrm{v}$ DMSO) (a), or $25 \mathrm{nM}$ siRNA to JAM-A or siNEG control (b). Cell extracts were prepared after $24 \mathrm{~h}$ of treatment or $72 \mathrm{~h}$ of gene silencing and immunoblotted for p-ERK1/2, Total ERK1/2, p-c-FOS-T232 and $\beta$-actin. Experiments were repeated three times and representative blots are shown.

investigated in TNBC cells. As shown in Figure 3b, a significant increase in dual positivity for Annexin- $\mathrm{V}$ and PI in $4 \mathrm{~T} 1$ cells suggested that Tetrocarcin-A drove cells into late apoptosis. Furthermore, a significant enhancement in mitochondrial membrane permeability upon Tetrocarcin-A treatment of 4T1 cells (Figure 3c) was consistent with the induction of apoptosis through a mitochondrial-dependent intrinsic pathway. Caspase-dependency of this process was next evidenced by reductions in the pro-forms of capsases9 and -3 following Tetrocarcin-A treatment (Figure 3d); in addition to a reversal of PI-positivity in $4 \mathrm{~T} 1$ cells in the presence of the pan-caspase inhibitor ZVAD-FMK (16) (Figure 3e).
Tetrocarcin-A inhibits growth of primary TNBC cells and inhibits the semi-in vivo growth of TNBC tumor xenografts. Finally, the relevance of these findings to in vivo conditions was examined. TNBC primary cells derived from breast cancer patients were sensitive to the anti-growth effects of Tetrocarcin-A after $24 \mathrm{~h}$ (Figure 4a). Furthermore, in an in ovo/semi-in vivo chicken egg chorioallantoic membrane (CAM) tumor model, previously validated for the preclinical evaluation of anti-cancer agents $(9,10)$, Tetrocarcin-A reduced the gross size of $4 \mathrm{~T} 1 \mathrm{TNBC}$ cell xenografts (Figure 4b). This corresponded microscopically to widespread cell death and significantly increased positivity for cleaved caspase-3 in tumor cells ( $\sim 60 \%$ in treated cells versus $\sim 10 \%$ 
a

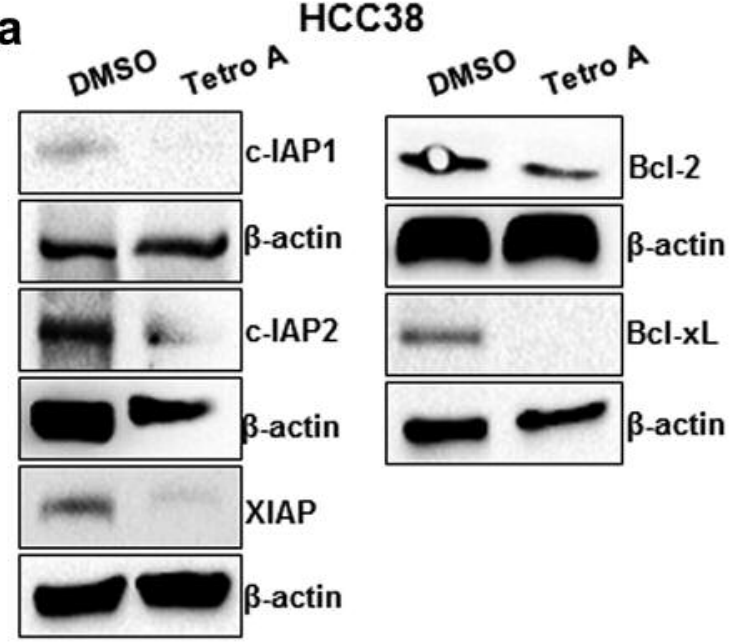

b

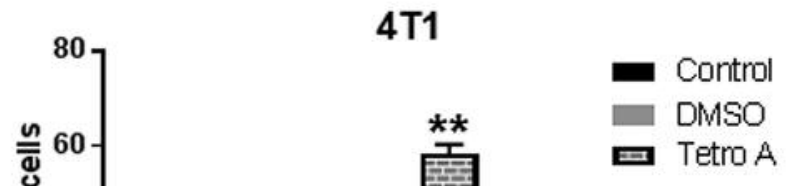

C

4T1

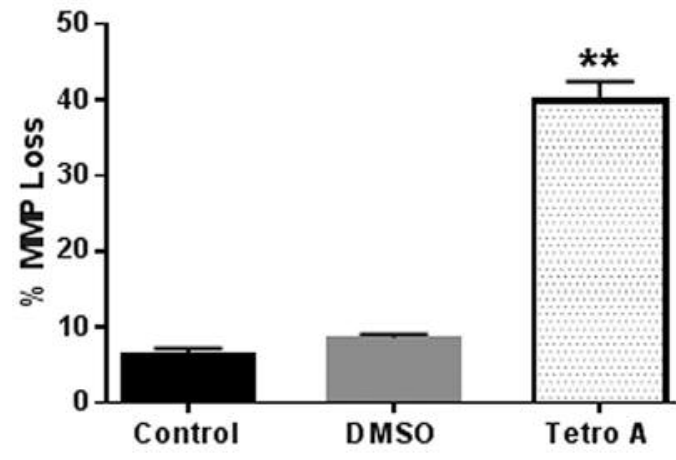

d

4T1

24h

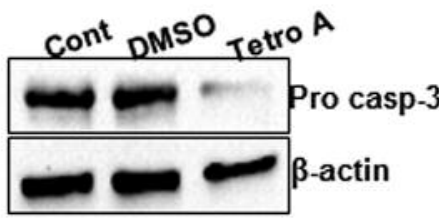

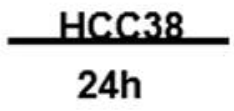

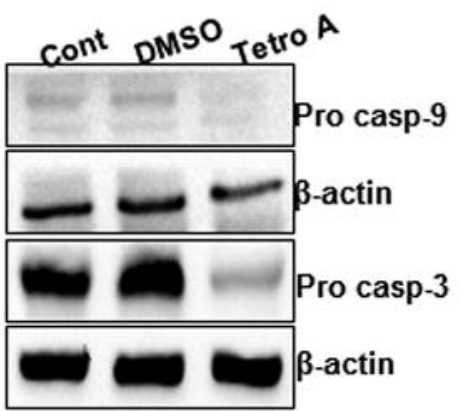

e

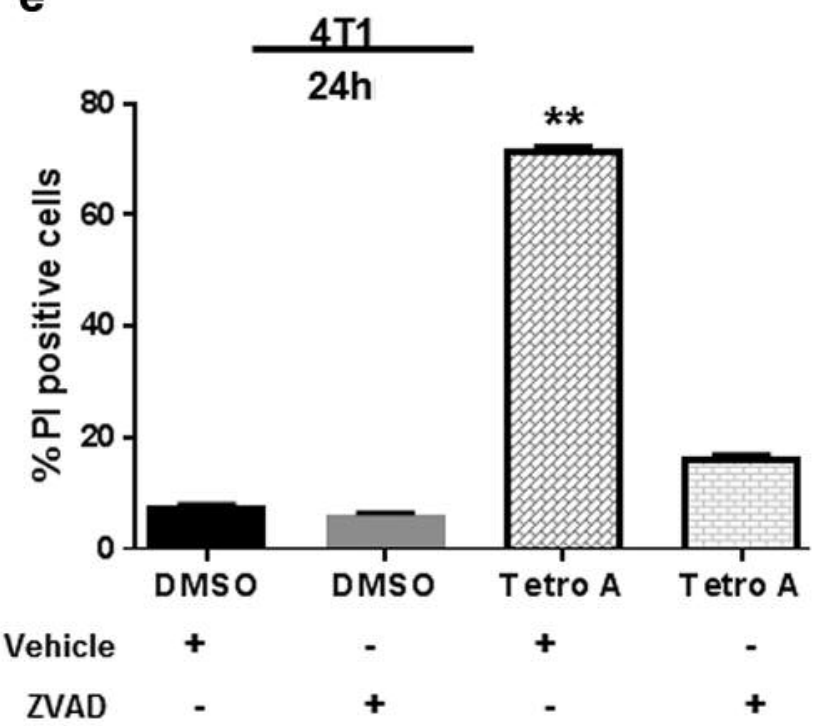

Figure 3. Tetrocarcin-A induced apoptosis in TNBC cells by activating caspases and down-regulating anti-apoptotic proteins. (a) HCC 38 cells were treated for $24 \mathrm{~h}$ with $2.5 \mu \mathrm{M}$ Tetrocarcin-A or vehicle $(0.01 \% \mathrm{v} / \mathrm{v}$ DMSO), and extracts were immunoblotted for c-IAP-1, XIAP, c-IAP-2, Bcl-2, Bcl-xL or $\beta$-actin. (b) $4 T 1$ cells were treated with $2.5 \mu M$ Tetrocarcin-A or vehicle $(0.01 \%$ v/v DMSO), stained for Annexin V/propidium iodide (PI) and flow cytometrically analyzed. (c) $4 T 1$ cells treated with $2.5 \mu \mathrm{M}$ Tetrocarcin-A or vehicle (0.01\% v/v DMSO) were stained with TMRM and flow cytometrically analyzed.(d) 4 T1 or HCC38 cells were treated for $24 \mathrm{~h}$ with $2.5 \mu \mathrm{M}$ Tetrocarcin-A or vehicle $10.01 \%$ v/v DMSO) and cell extracts analyzed by western blotting for caspase-3, caspase-9 and $\beta$-actin. (e) $4 T 1$ cells were treated for $1 \mathrm{~h}$ with $2.5 \mu \mathrm{M}$ Tetrocarcin-A or vehicle $(0.01 \% \mathrm{v} / \mathrm{v}$ DMSO) then further incubated with ZVAD-FMK $(50 \mu M)$ or vehicle $(0.3 \%$ v/v DMSO) and stained with PI. Error bars reflect SEM of triplicate experiments $(* * p<0.005$, two-tailed unpaired student's t-test; vehicle versus Tetrocarcin-A). 


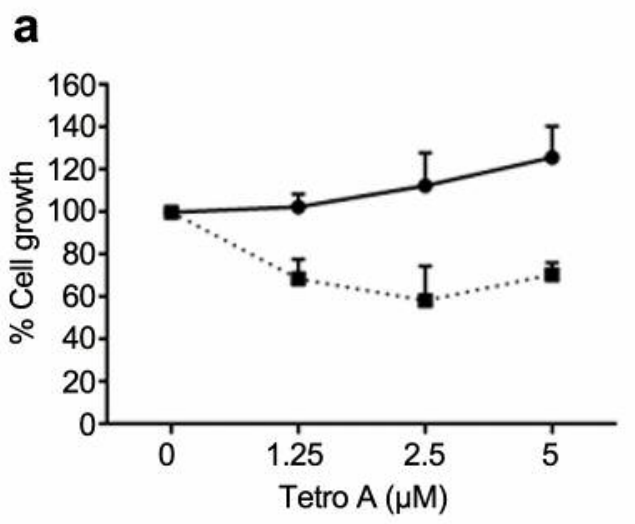

b

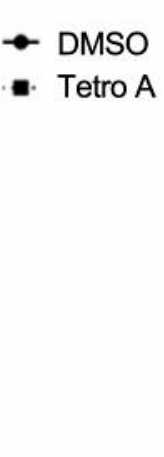

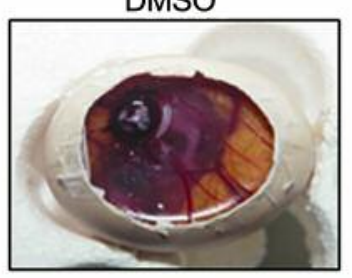

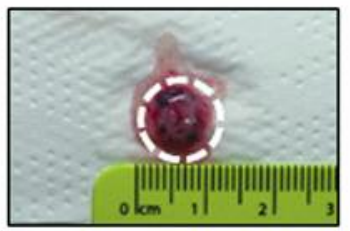

Tetro A
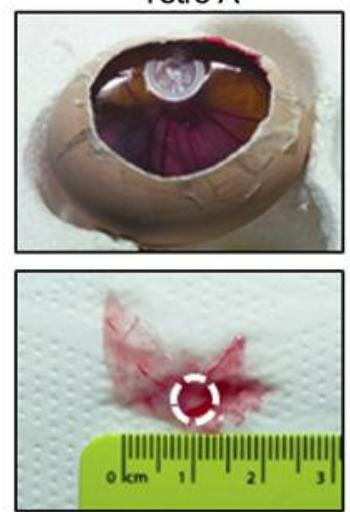

2

3

4
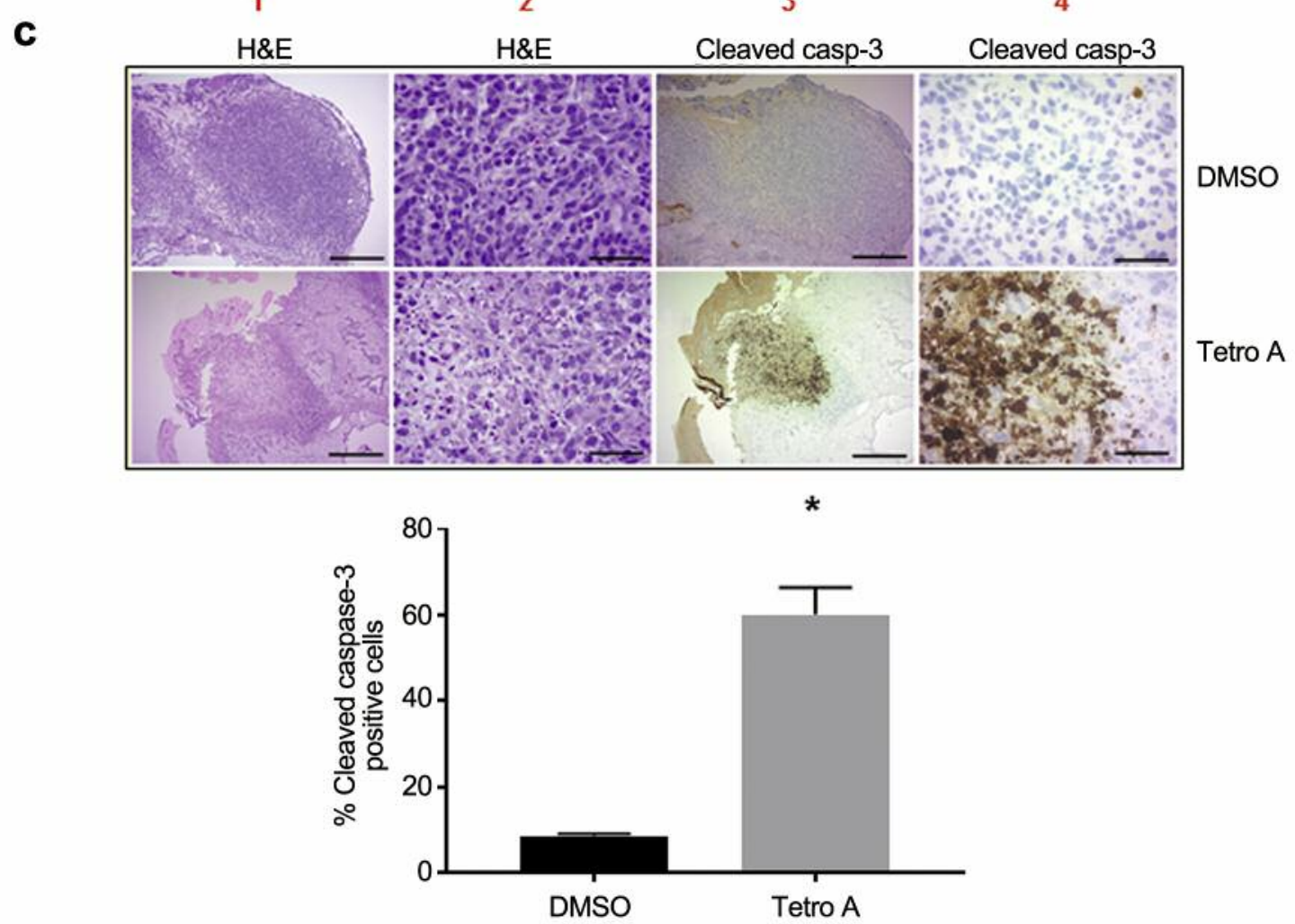

Figure 4. Tetrocarcin-A was cytotoxic to human primary TNBC cells and inhibited semi-in vivo growth of TNBC xenografts on the chorionic allantoic membrane. (a) TNBC primary breast cancer cells were treated for $48 \mathrm{~h}$ with Tetrocarcin-A or vehicle (0.01-0.006\% v/v DMSO) and cell viability was measured. (b) $4 T 1$ cells xenografted onto the chicken egg CAM were treated with $2.5 \mu M$ Tetrocarcin-A (or vehicle) then sectioned and stained for cleaved caspase-3 (c). Representative photomicrographs of TNBC cells infiltrating the CAM (control; top). Panels 1, 2 show H\&E staining; Panels 3, 4 show cleaved caspase-3 (brown) counterstained with hematoxylin. Scale bars are 250 um for panels 1, 2; 25 um for panels 3, 4). Error bars refer to SEM of triplicate experiments $\left({ }^{*} p<0.05\right.$; by two-tailed unpaired student's $t$-test; comparing vehicle controls versus Tetroarcin-A-treated tumors). The CAM assay was repeated twice, with multiple eggs per iteration.

in controls; Figure 4c). Taken together, our results are consistent with a model, whereby Tetrocarcin-A causes down-regulation of JAM-A and induces apoptosis in TNBC via a pathway involving c-FOS-mediated regulation of IAPs.

\section{Discussion}

Triple-negative breast cancers (TNBC) present significant clinical challenges, as they lack traditional cell surface 
markers both for aiding early diagnosis and for developing targeted therapies. TNBC patients typically have shorter overall survival with an early peak of distant recurrences just 3 years after diagnosis (17). We have previously reported that Crassin, a natural compound derived from coral, causes cytostasis in TNBC cells (18). We have also identified another natural compound, Tetrocarcin-A, which induces apoptosis in hormone receptor-positive breast cancer models (4). Focussing upon TNBC models in the current study, Tetrocarcin-A was found to down-regulate the protein expression of JAM-A, pERK1/2 and p-c-FOS; which in turn down-regulates the inhibitor of apoptosis (IAP) proteins and induces apoptotic cell death in TNBC cells.

Several lines of evidence support the likelihood of a central role for JAM-A down-regulation in the anti-viability mechanism of Tetrocarcin-A. First, Tetrocarcin-A significantly reduced JAM-A protein expression; and its anti-growth effects on TNBC cells were phenocopied by direct gene silencing of JAM-A. Notably, JAM-A has recently been described as important for self-renewal in TNBC cancer stem cells (19). Second, stable overexpression of JAM-A in hormone receptorpositive breast cancer cells has recently been shown to offer some protection against the anti-viability effects of Tetrocarcin-A (4). Third, phosphorylation of the MAPK survival effectors ERK1/2 was similarly reduced by either Tetrocarcin-A treatment or JAM-A gene silencing. Fourth, the levels of Threonine-232-phosphorylated c-FOS were reduced by either Tetrocarcin-A treatment or JAM-A gene silencing. ERK has been shown to transcriptionally activate the cell fate regulator c-FOS by phosphorylating it on Threonine-232 (14), therefore it is suggested that JAM-A down-regulation induced by Tetrocarcin-A exerts downstream signals through this mechanism. It is, however, interesting to note that Tetrocarcin$\mathrm{A}$ induced greater reductions in pERK and p-c-FOS levels than JAM-A silencing alone. Although this may simply reflect varying degrees of JAM-A loss, the possibility that Tetrocarcin-A impacts the same signaling effectors by JAMA-independent mechanisms cannot be excluded.

While the transcriptional targets of c-FOS are numerous, the IAP family represents an important subset in the context of cell fate regulation. Accordingly, Tetrocarcin-A-induced reductions in the expression levels of several IAPs are likely to be central to its mechanism of action. In the TNBC models used, this translated into removal of a physiological block on apoptosis, and activation of caspase-dependent apoptosis via the intrinsic pathway.

In conclusion, it is shown for the first time that the natural antibiotic Tetrocarcin-A exerts pro-apoptotic effects on TNBC cells involving sequential down-regulation of JAMA, pERK1/2, p-c-FOS and IAPs. An in ovo rather than a murine xenograft model was used for substantiation of these results, since the former has been well-validated for cancer drug testing $(9,10)$ and did not require an animal licence under the selected conditions. Reports have indicated an inverse link between JAM-A expression and survival in breast cancer patient populations as a whole (2, 3, 20); and suggested JAM-A antagonism as a promising strategy. Our data further propose that JAM-A targeting has particular value in TNBC, potentially presenting a novel drug in a limited therapeutic landscape. Given a current lack of JAMantagonists on the market, the capacity of Tetrocarcin-A to down-regulate JAM-A merits investigation for oncology applications.

\section{Conflicts of Interest}

The Authors have no conflicts of interest to disclose regarding this study.

\section{Authors' Contributions}

SHV and AMH designed the study and wrote the manuscript; SHV performed most of the experimental work and data analysis; RGBC, CER, YES and LH performed some experimental work; HJ analyzed and quantified the outputs of the CAM assay. All Authors read and approved the final manuscript.

\section{Acknowledgements}

This research was funded by Science Foundation Ireland (SFI; grant \# 13/IA/1994 to AMH). RGBC was funded by CAPES, Coordination for the Improvement of Higher Education Personnel - Brazil (Science without Borders Programme - Process 013306/2013-08). YES was funded by the Health Research Board of Ireland (HRA-POR-2014-545 to AMH). The Authors thank the NCI/DTP Open Chemical Repository (https://dtp.cancer.gov) for natural compound NSC 333856 (Tetrocarcin-A); Dr. Brona Murphy for the cIAP-1 antibody, Shannonvale Hatchery (Ireland) for fertilized chicken eggs and Mr. Ray Lohan for egg photography.

\section{References}

1 Song W, Hwang Y, Youngblood VM, Cook RS, Balko JM, Chen $\mathrm{J}$ and Brantley-Sieders DM: Targeting EphA2 impairs cell cycle progression and growth of basal-like/triple-negative breast cancers. Oncogene 36: 5620-5630, 2017. PMID: 28581527, DOI: $10.1038 /$ onc.2017.170.

2 McSherry EA, McGee SF, Jirstrom K, Doyle EM, Brennan DJ, Landberg G, Dervan PA, Hopkins AM and Gallagher WM: JAM-A expression positively correlates with poor prognosis in breast cancer patients. Int J Cancer 125: 1343-1351, 2009. PMID: 19533747, DOI: 10.1002/ijc.24498.

3 Murakami M, Giampietro C, Giannotta M, Corada M, Torselli I, Orsenigo F, Cocito A, d'Ario G, Mazzarol G, Confalonieri S, Di Fiore PP and Dejana E: Abrogation of junctional adhesion molecule-A expression induces cell apoptosis and reduces breast cancer progression. PLoS One 6: e21242, 2011. PMID: 21695058, DOI: 10.1371/journal.pone.0021242.

4 Vellanki SH, Cruz RGB, Jahns H, Hudson L, Sette G, Eramo A and Hopkins AM: Natural compound Tetrocarcin-A downregulates 
Junctional Adhesion Molecule-A in conjunction with HER2 and inhibitor of apoptosis proteins and inhibits tumor cell growth. Cancer Lett 440-441: 23-34, 2019. PMID: 30312728, DOI: 10.1016/j.canlet.2018.09.032.

5 Kobinata K, Uramoto M, Mizuno $\mathrm{T}$ and Isono $\mathrm{K}$ : A new antibiotic, antlermicin A. J Antibiot (Tokyo) 33: 244-246, 1980. PMID: 7380735.

6 Nakajima H, Sakaguchi K, Fujiwara I, Mizuta M, Tsuruga M, Magae J and Mizuta N: Apoptosis and inactivation of the PI3kinase pathway by tetrocarcin A in breast cancers. Biochem Biophys Res Commun 356: 260-265, 2007. PMID: 17350598, DOI: $10.1016 /$ j.bbrc.2007.02.136.

7 Nakashima T, Miura M and Hara M: Tetrocarcin A inhibits mitochondrial functions of $\mathrm{Bcl}-2$ and suppresses its antiapoptotic activity. Cancer Res 60: 1229-1235, 2000. PMID: 10728681, DOI: 10728681.

8 Donatello S, Hudson L, Cottell DC, Blanco A, Aurrekoetxea I, Shelly MJ, Dervan PA, Kell MR, Stokes M, Hill AD and Hopkins AM: An imbalance in progenitor cell populations reflects tumour progression in breast cancer primary culture models. J Exp Clin Cancer Res 30: 45, 2011. PMID: 21521500 , DOI: 10.1186/1756-9966-30-45.

9 Stupack DG, Teitz T, Potter MD, Mikolon D, Houghton PJ, Kidd VJ, Lahti JM and Cheresh DA: Potentiation of neuroblastoma metastasis by loss of caspase-8. Nature 439: 95-99, 2006. PMID: 16397500, DOI: 10.1038/nature04323.

10 Unterkircher T, Cristofanon S, Vellanki SH, Nonnenmacher L, Karpel-Massler G, Wirtz CR, Debatin KM and Fulda S: Bortezomib primes glioblastoma, including glioblastoma stem cells, for TRAIL by increasing tBid stability and mitochondrial apoptosis. Clin Cancer Res 17: 4019-4030, 2011. PMID: 21525171, DOI: 10.1158/1078-0432.CCR-11-0075.

11 Goetsch L, Haeuw JF, Beau-Larvor C, Gonzalez A, Zanna L, Malissard M, Lepecquet AM, Robert A, Bailly C, Broussas M and Corvaia N: A novel role for junctional adhesion molecule$\mathrm{A}$ in tumor proliferation: modulation by an anti-JAM-A monoclonal antibody. Int J Cancer 132: 1463-1474, 2013. PMID: 22886345, DOI: $10.1002 /$ ijc. 27772 .

12 Lanczky A, Nagy A, Bottai G, Munkacsy G, Szabo A, Santarpia L and Gyorffy B: miRpower: a web-tool to validate survivalassociated miRNAs utilizing expression data from 2178 breast cancer patients. Breast Cancer Res Treat 160: 439-446, 2016. PMID: 27744485, DOI: 10.1007/s10549-016-4013-7.
13 Gyorffy B, Lanczky A, Eklund AC, Denkert C, Budczies J, Li Q and Szallasi Z: An online survival analysis tool to rapidly assess the effect of 22,277 genes on breast cancer prognosis using microarray data of 1,809 patients. Breast Cancer Res Treat 123: 725-731, 2010. PMID: 20020197, DOI: 10.1007/s 10549-009-0674-9.

14 Monje P, Hernandez-Losa J, Lyons RJ, Castellone MD and Gutkind JS: Regulation of the transcriptional activity of c-Fos by ERK. A novel role for the prolyl isomerase PIN1. J Biol Chem 280: 3508135084, 2005. PMID: 16123044, DOI: 10.1074/jbc.C500353200.

15 Monje P, Marinissen MJ and Gutkind JS: Phosphorylation of the carboxyl-terminal transactivation domain of c-Fos by extracellular signal-regulated kinase mediates the transcriptional activation of AP-1 and cellular transformation induced by platelet-derived growth factor. Mol Cell Biol 23: 7030-7043, 2003. PMID: 12972619.

16 Vellanki SH, Grabrucker A, Liebau S, Proepper C, Eramo A, Braun V, Boeckers T, Debatin KM and Fulda S: Small-molecule XIAP inhibitors enhance gamma-irradiation-induced apoptosis in glioblastoma. Neoplasia 11: 743-752, 2009. PMID: 19649204.

17 Collignon J, Lousberg L, Schroeder H and Jerusalem G: Triplenegative breast cancer: treatment challenges and solutions. Breast Cancer (Dove Med Press) 8: 93-107, 2016. PMID: 27284266, DOI: 10.2147/BCTT.S69488.

18 Richards CE, Vellanki SH, Smith YE and Hopkins AM: Diterpenoid natural compound C4 (Crassin) exerts cytostatic effects on triple-negative breast cancer cells via a pathway involving reactive oxygen species. Cell Oncol (Dordr) 41: 3546, 2018. PMID: 29134467, DOI: 10.1007/s 13402-017-0357-1.

19 Thiagarajan PS, Hitomi M, Hale JS, Alvarado AG, Otvos B, Sinyuk M, Stoltz K, Wiechert A, Mulkearns-Hubert E, Jarrar A, Zheng Q, Thomas D, Egelhoff T, Rich JN, Liu H, Lathia JD and Reizes O: Development of a fluorescent reporter system to delineate cancer stem cells in triple-negative breast cancer. Stem Cells 33: 21142125, 2015. PMID: 25827713, DOI: 10.1002/ stem.2021.

20 Brennan K, McSherry EA, Hudson L, Kay EW, Hill AD, Young LS and Hopkins AM: Junctional adhesion molecule-A is coexpressed with HER2 in breast tumors and acts as a novel regulator of HER2 protein degradation and signaling. Oncogene 32: 27992804, 2013. PMID: 22751120, DOI: 10.1038/onc.2012.276.

Received January 18, 2019

Revised February 1, 2019

Accepted February 5, 2019 